\title{
P2X7R antagonists in chronic stress-based depression models: a review
}

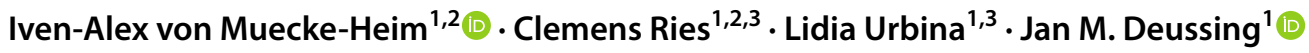

Received: 1 April 2021 / Accepted: 12 July 2021 / Published online: 19 July 2021

(c) The Author(s) 2021

\begin{abstract}
Depression affects around 320 million people worldwide. Growing evidence proposes the immune system to be the core interface between psychosocial stress and the neurobiological and behavioural features of depression. Many studies have identified purinergic signalling via the $\mathrm{P} 2 \mathrm{X} 7$ receptor $(\mathrm{P} 2 \mathrm{X} 7 \mathrm{R})$ to be of great importance in depression genesis yet only a few have evaluated P2X7R antagonists in chronic stress-based depression models. This review summarizes their findings and analyses their methodology. The four available studies used three to nine weeks of unpredictable, chronic mild stress or unpredictable, chronic stress in male mice or rats. Stress paradigm composition varied moderately, with stimuli being primarily psychophysical rather than psychosocial. Behavioural testing was performed during or after the last week of stress application and resulted in depressive-like behaviours, immune changes (NLRP3 assembly, interleukin-1 $\beta$ level increase, microglia activation) and neuroplasticity impairment. During the second half of each stress paradigm, a P2X7R antagonist (Brilliant Blue G, A-438079, A-804598) was applied. Studies differed with regard to antagonist dosage and application timing. Nonetheless, all treatments attenuated the stress-induced neurobiological changes and depressive-like behaviours. The evidence at hand underpins the importance of P2X7R signalling in chronic stress and depression. However, improvements in study planning and reporting are necessary to minimize experimental bias and increase data purview. To achieve this, we propose adherence to the Research Domain Criteria and the STRANGE framework.
\end{abstract}

Keywords Animal models $\cdot$ Chronic stress $\cdot$ Microglia and macrophages $\cdot \mathrm{P} 2 \mathrm{X} 7$ receptor $(\mathrm{P} 2 \mathrm{X} 7 \mathrm{R}) \cdot \mathrm{P} 2 \mathrm{X} 7 \mathrm{R}$ antagonists

\section{Introduction}

Depression is characterised by three main symptoms: low mood, anhedonia, and decreased energy [1]. Approximately 320 million or $4.4 \%$ of the global population are affected [2]. Women have a twofold increased disease risk compared to men [3]. Depression sits among the top three causes of years lived with disability worldwide [4] and burdens individual life prospects and health care systems alike [5-7].

Iven-Alex von Muecke-Heim

iven_muecke-heim@psych.mpg.de

Jan M. Deussing

deussing@psych.mpg.de

1 Max Planck Institute of Psychiatry, Molecular Neurogenetics, Munich, Germany

2 International Max Planck Research School for Translational Psychiatry (IMPRS-TP), Munich, Germany

3 Graduate School of Systemic Neurosciences, University of Munich (LMU), Munich, Germany
Treatment employed depends on depression severity and patient response, yet overall yields heterogeneous outcomes $[8,9]$. In fact, only $50-70 \%$ of depressed patients recover within 1 year of diagnosis $[6,10]$ and an alarming $15-20 \%$ experience a chronic course of depression ( $>24$ months) $[6,10,11]$. The latter is associated with reduced quality of life, limitations of daily activities, treatment resistance, suicide attempts and comorbidities [12]. Aside the serendipitous discovery of ketamine, no other major therapeutic breakthroughs have been achieved in the past decades [13]. Moreover, no reliable and clinically applicable diagnosis or outcome prediction tools are available $[8,14]$. This dire situation urges us to evaluate and optimize preclinical and clinical methods to advance novel therapeutic strategies [9].

Within recent years, the concept of depression has changed and now more than ever includes immunological disease features [15-18]. However, on a mere environmental level, psychosocial stress is still the most common and major risk factor of depression [14, 19-25]. Concerning the neurobiological link between stress and depression, several studies 
have highlighted the role of microglia and macrophage (M/ Ms) activation [18, 26-32] mediated by purinergic signalling via the membrane-bound adenosine triphosphate (ATP) receptor P2X7 (P2X7R) [33-40]. On a genetic level, studies in humans and mice have found an association between polymorphisms in the $P 2 R X 7$ gene and depressive symptoms [41-46]. Meanwhile, a growing body of evidence emphasizes the potential of P2X7R as a novel drug target in depression [33, 35, 47-51] and small, placebo-controlled phase II studies investigating P2X7R antagonists in major depressive disorder are ongoing [52,53]. Yet, only a handful of studies have evaluated P2X7R antagonistic drugs in chronic stressbased depression models [34].

Based on the emerging role of P2X7R as an interface between stress and the immunological features of depression, this review aims to analyse the available studies on pharmacological P2X7R antagonism in translational murine depression models. Although no single approach can represent the plethora of environmental and biological factors of depression, chronic stress-based models are currently considered the most valid option in reflecting disease complexity overall. They reliably induce core depressive symptoms, behaviours and biological changes by exposing animals to unpredictable, mild to moderate environmental stress over several days to weeks. Because of that, they are common models in translational neuropsychiatry [13, 54-56]. We thus included studies using chronic psychosocial and psychophysical stress (CPSS/CPPS) in the form of chronic unpredictable stress (CUS), unpredictable, chronic mild stress (UCMS/CMS) or chronic social defeat (CSD) in this review. After summarizing the evidence revolving around chronic stress, the immune system and P2X7R signalling in depression, we examine the available studies from which we can draw meaningful insights for future research.

\section{From stress to depression: the emerging role of the immune system}

The pathogenesis of depression is complex and has been extensively investigated. Nowadays, explanatory approaches integrate the entirety of endocrine, neurochemical, and plasticity aberrations in a fine-grained framework of environmental influences and epi-/genetic as well as psychosocial vulnerabilities $[14,34,57]$. Since the concept of stress was introduced to the scientific community in 1936 by Hans Selye [58, 59], a strong link between CPSS/CPPS and depression has been reported. Many studies and metaanalyses have demonstrated an elevated depression risk following childhood maltreatment and adverse life events in adolescence and adulthood [21,60-66]. The biological mechanisms that translate this form of environmental stress into the nosological entity called depression are not yet fully understood, but the immune system, in particular cytokines and microglia, is proposed to be a principal component [16, 35, 67-69]. This notion stems from a larger number of human and rodent studies, which found elevated levels of inflammatory markers including but not limited to C-reactive protein (CRP), interleukin-1-family cytokines like interleukin- 1 beta (IL-1 $\beta$ ) and IL-18, tumour necrosis factor alpha (TNF- $\alpha$ ) and IL-6 in CPSS/CPPS or depression $[15,70-78]$.

Interestingly and in line with this so-called cytokine hypothesis of depression [16], immunological diseases like asthma or diabetes are associated with elevated inflammatory cytokine levels and an up to twofold increased depression risk $[68,79-82]$. On a genetic level, a recent correlation study based on a large genome-wide association study reported an overlap between CRP levels and depression symptoms as well as an association of upregulated IL-6 with suicidality [72]. In 2014, a clinical study found elevated NLR family pyrin domain containing 3 (NLRP3) and caspase- 1 gene expression in the blood of $n=40$ treatmentnaive depressed patients. The NLRP 3 and caspase- 1 expression along with IL-1 $\beta$ and IL-18 levels were ameliorated by amitriptyline treatment [83]. NLRP3 inflammasome assembly, caspase- 1 activation and IL- $1 \beta$ release are generally known to be triggered by P2X7R activation, which is one of the main NLRP3 activators [36] and a potent inductor of M/Ms activation and proliferation [84, 85]. Accordingly, animal studies investigating acute and chronic stress in the context of depressive-like states reported altered recruitment and increased activation of $\mathrm{M} / \mathrm{Ms}$, predominantly in frontolimbic regions [27, 86-95]. In humans, positron emission tomography studies in depressed patients detected elevated microglia activity in the prefrontal cortex (PFC), anterior cingulate cortex and insula by using a radiotracer for translocator protein [96-98], a transmembrane protein located in the outer mitochondrial membrane that serves as a sensitive neuroinflammation marker [99]. Overall, these findings suit the recent notion of depression as a microgliopathy [26]. It is even discussed whether M/Ms are the key facilitators of the relation between CPSS/CPPS and depression $[15,18,100]$. In line with this, several studies have demonstrated the joint role of IL- $1 \beta$, IL- 6 and TNF- $\alpha$ along with M/Ms activation in tryptophan-kynurenine-pathway disruption, glutamate excitotoxicity, blood brain barrier disruption and neuronal loss in depressive and suicidal behaviour [101-104]. Based on these findings, the P2X7R-NLRP3-IL- $1 \beta$ cascade is proposed to be the primary interface between CPSS/CPPS, humoral and cellular immunity, and depression [15-17, $33-35,38,67,94,100,105]$. 


\section{Neuroinflammation, P2X7R-signalling and chronic stress}

Purinergic signalling is a phylogenetically ancient, ubiquitous cellular mechanism involved in cell-to-cell crosstalk, tissue homeostasis and immune functioning [36, 106]. Nearly all cells release purines and bind them with a variety of membrane-bound receptors. The purine receptor family consists of $\mathrm{P} 1$ receptors for adenosine and $\mathrm{P} 2$ receptors for nucleotides. The latter are again divided into eight metabotropic P2Y and seven ligand-gated ionotropic P2X receptors $[36,107]$. The contribution of $\mathrm{P} 2 \mathrm{X} 7 \mathrm{R}$ signalling in sterile inflammation and infection has been examined in systemic and brain-specific entities like Parkinson's or Alzheimer's disease, bipolar disorder, and schizophrenia [36, 38, 43, 108, 109]. At the pathogenetic intersection of chronic stress and depression, P2X7R signalling has been identified as a pertinent factor in causing the numerous and complex neurobiological aberrations [33, 34, 39, 43, 48].

In the brain, P2X7R is strongly expressed on M/Ms $[30,110,111]$ and mainly active in chronic, inflammatory conditions due to its high activation threshold and slow desensitization (half maximal effective ATP concentration: 2-4 micromolar) compared to other P2X receptors [36, $109,111,112]$. The functional P2X7R is comprised of three congregating P2X7R monomers [36]. In the context of neuropsychiatric research, many studies have found that CPSS/CPPS intensifies glutamate release from neurons, which in return triggers more ATP release from neurons, astrocytes, and microglia [33, 35, 51, 67, 94, 113-116]. In the extracellular space, ATP is a part of damage-associated molecular patterns, which act as molecular distress signals $[117,118]$. Following ATP binding, P2X7R allows immediate potassium efflux, sodium and calcium influx and the passage of several large organic cations via an intrinsic macropore function [36, 47, 117]. The long-standing hypothesis of P2X7R pore dilation was recently disproved [36, 117, 119, 120]. Upon sustained P2X7R activation, for example by CPSS/CPPS, apoptosis or necrosis is triggered by caspase- 3 cleavage $[34,47,117]$.

Intracellularly, signal transduction is facilitated by the P2X7R-induced potassium concentration decline. This induces the NIMA-related kinase 7 (NEK7) dependent assembly of the NLRP3 inflammasome [121], a multiprotein complex causing caspase- 1 activation and the release of proinflammatory cytokines like IL-1 $\beta$ and IL-18 from astrocytes and microglia [38, 94, 109, 122, 123]. In addition, calcium influx upregulates the cellular energy metabolism by stimulating glycolysis as well as oxidative phosphorylation and causes further glutamate and ATP release from microglia and astrocytes, leading to heightened excitotoxicity $[34,36]$. Aside from this, P2X7R activates several other inflammatory pathways including nuclear factor 'kappa-light-chain-enhancer' of activated B-cells (NFKB), which jointly cause increased pro-IL-1 $\beta$ and pro-IL-18 transcription as well as the production of IL-6, IL- $1 \alpha$ and TNF- $\alpha[109,123]$. The P2X7R mediated IL-1 $\beta$ release also induces an array of inflammatory effector molecules including cyclooxygenases, eicosanoids and reactive oxygen as well as nitrogen species $[34,109,124$, 125]. The pathways following P2X7R stimulation in M/Ms are illustrated in Fig. 1. Taken together, the multiple cellular and humoral immune processes downstream of P2X7R may translate CPSS/CPPS into the hallmark symptoms and neurobiological aberrations of depression [15-17, 33-35, $76,116,126-128]$.

\section{The P2X7R-NLRP3-IL-1 $\beta$ cascade mediates depressive-like behaviour}

To support the importance of the P2X7R-NLRP3-IL-1 $\beta$ cascade in mediating depressive-like behaviour, we present the evidence for each single pathway component.

Chronic (21 days) bilateral, stereotactic injection of ATP or 2'(3')-O-(4-benzoylbenzoyl)-ATP (BzATP) into the hippocampus of male Sprague-Dawley rats led to depressivelike behaviour in the form of reduced rearing times and total distance travelled in the open field test (OFT) and decreased struggling and increased immobility in the tail suspension test (TST). Correspondingly, P2X7R knockout caused stressresilience in the forced swim test (FST) and OFT compared to C57BL/6 mice when exposed to a 5-week CUS regime [113]. Basso et al. [129] also found antidepressant-like effects of P2X7R inactivation in the FST and TST. In a 12-week UCMS model in male Wistar rats, Pan et al. [130] observed a significant upregulation of NLRP3 and NFKB signalling along with increased IL-1 $\beta$ serum und PFC levels. Wang et al. [88] performed a similar UCMS paradigm in male Sprague-Dawley rats and reported depressive-like behaviour in the form of decreased struggling in the FST, reduced rearing and locomotor activity in the OFT and decreased open arm time in the elevated plus maze (EPM). In a related approach, Zhang et al. [131] demonstrated that a pre-treatment of 8-week-old, male BALB/c mice with the NLRP3 inhibitor VX-765 inhibited the development of depressive-like behaviours. Compared to untreated but stressed controls, the VX-765 group showed no changes in the sucrose preference test (SPT) nor TST after 4 weeks of UCMS. Serum and hippocampal IL-1 $\beta$ levels as well as NLRP3 and caspase-1 measures remained unaltered in the VX-765 UCMS group. Similar effects were observed following 30 days of immobilisation stress in male NLRP3-null C57BL/6 mice. These animals did not show ATP or IL-1 $\beta$ level increase in the PFC or hippocampus and social interaction, food intake as well as performance in the 


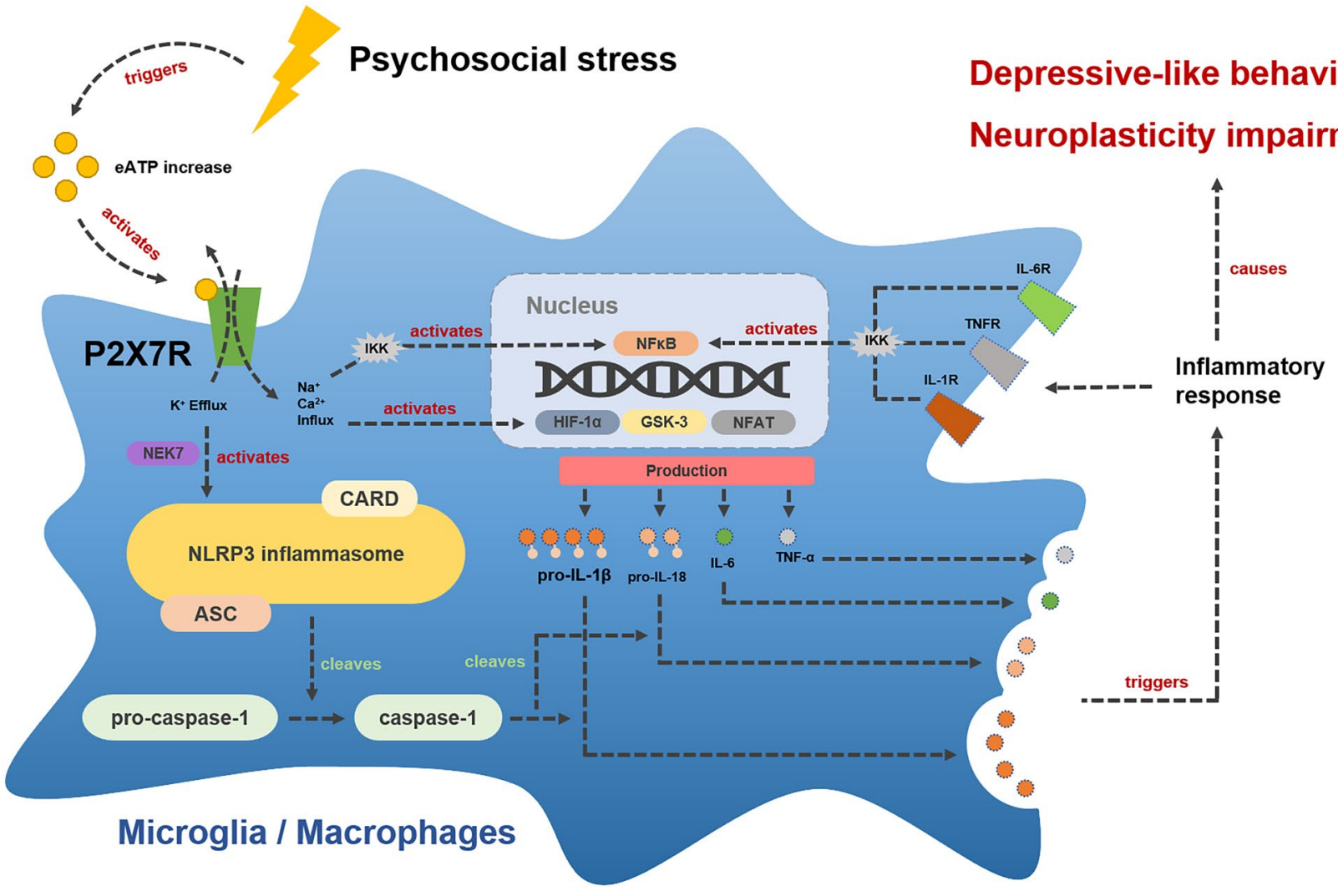

Fig. 1 Hypothesized inflammatory pathways downstream of P2X7R in $\mathrm{M} / \mathrm{Ms}$ following psychosocial stress. Upon psychosocial stress exposure, neurons and astrocytes release ATP into the extracellular space (eATP). This activates P2X7R on microglia and macrophages and causes NLRP3 assembly, caspase-1 activation and NFKB upregulation. These mechanisms jointly cause increased IL-1 $\beta$, IL-18, IL-6 and TNF- $\alpha$ production and release. Ultimately, this leads to a cellular and humoral inflammatory response, neuroplasticity impairment

SPT and FST remained unaltered [116]. Meanwhile, injection of recombinant IL-1 $\beta$ into the ventricle of Sprague-Dawley rats caused decreased sucrose intake in the SPT, increased immobility in the TST and supressed social interaction [132]. Vice versa, IL-1 $\beta$ antibody treatment during a 2-week CUS regime prevented depressive-like behaviour in the SPT and EPM compared to control mice receiving unspecific immunoglobulins (IgG) [94]. Analogously, 5 weeks of UCMS failed to induce behavioural or endocrine changes in male, IL-1 receptor knockout mice, while the same UCMS reduced neurogenesis, decreased sucrose preference in the SPT and lowered social exploration in C57BL/6 controls [133]. In line with these findings, intraventricular TNF- $\alpha$ injection induced depressive-like behaviour measured in the FST and TST in 6-week old, male C57BL/6 mice [134], while chronic administration of a TNF- $\alpha$ inhibitor (Infliximab $5 \mathrm{mg} / \mathrm{kg} /$ week) was able to attenuate depressive-like behaviour in the FST, EPM and SPT after 8 weeks of UCMS [56, 135]. TNF receptor 1 knockout mice also showed significantly decreased despair and depressive-like behaviour. NEK7 NIMA-related kinase protein 7, CARD caspase activation and recruitment domain, ASC apoptosis-associated speck-like protein containing a CARD, $N F k B$ nuclear factor 'kappa-light-chain-enhancer' of activated B-cells, IKK ІкBkinase-complex, HIF- $\alpha$ hypoxia-inducible factor 1-alpha, GSK-3 glycogen synthase kinase 3, NFAT nuclear factor of activated T-cells, $I L$ interleukin, $T N F-\alpha$ tumor necrosis factor alpha, cytokine receptor IL-R or TNFR

in the FST and TST compared to wild-type animals [134]. Last, application of an IL-6 antibody in male C57BL/6 mice exposed to 10 days of CSD reduced immobility in the TST and increased sucrose intake in the SPT [136]. Similarly, IL-6 knockout mice displayed reduced despair in the FST and TST, enhanced sucrose preference in the SPT and a partial resistance to learned helplessness compared to controls [137]. In summary, the evidence available demonstrates the importance of each component along the P2X7R-NLRP3-IL-1 $\beta$ cascade in mediating depressive-like behaviours and, when pharmacologically or genetically inactivated, in stress resilience. 


\section{P2X7R antagonists in murine chronic stress models}

We identified a total of four studies, namely Farooq et al. 2018, Aricioglu et al. 2009, Yue et al. 2017, and Iwata et al. 2016, which used P2X7R antagonists in CPSS/ CPPS-based murine models of depression (Table 1).

To induce depressive-like behaviour, two of the studies applied a UCMS paradigm, while the others used CUS. We could not find a single study that employed a CSD paradigm. The stimuli used in the different stress paradigms are almost identical, but reporting detail varied between studies. This was particulary true regarding the stimulus duration and the total amount of time animals were exposed to either one or several stimuli. All studies reported random stressor application and employed at least one stressor per day. Exclusively Farooq et al. 2018 gradually increased stressor density over the course of their 9 weeks UCMS paradigm to five applications per day. In regards to the stressfull stimuli and their composition, the studies partially overlap. All disrupted the circadian rhythm and applied cage shake, tilt or rotation. Three studies used temperature-based stressors and half-employed immobilsation or food and water deprivation. Stressors like tail nip or stroboscope application were only found in one study. Psychophysical stressors were predominant in all stress paradigms, while psychosocial ones like predator sounds, social isolation or crowding were used in only half the studies to a minor and negligible degree. Stress paradigm duration was $3,4,8$ or 9 weeks and thus differed between studies.

All studies exclusively included male animals. One study made use of BALB/cByJ mice, while the other three utilized either Sprague-Dawley or Wistar-Albino rats. Animal age at the start of UCMS or CUS varied between 7 and 10 weeks. However, Yue and Iwata et al. did not report the animals' age. Animal numbers per experimental group ranged between 7 and 15 .

In terms of P2X7R antagonist and dosage, Farooq and Aricioglu et al. injected Brilliant Blue G (BBG) intraperitoneal (i.p.) at $25-50 \mathrm{mg} / \mathrm{kg}$, while Yue et al. microinjected either BBG or A-438079 into both hippocampi. Exclusively Iwata et al. 2016 administered a novel, selective P2X7R antagonist called A-804598 i.p. with $5 \mathrm{mg} /$ $\mathrm{kg}$. Antagonist application timing and frequency differed between the studies, with a tendency towards a single administration per day in the second half of the respective stress regime.

Experimenter blinding was reported in all studies, however, varying in extent. Behavioural assessment was nonuniform among the studies: an overlap was only found for the FST and the SPT, which were applied in half the studies. Beyond this, the following tests were each applied in one single study: OFT, EPM, Coat State Score, Nest Building Score, Novelty Suppressed Feeding Test (NSFT). Throughout the studies, behavioural testing was performed after or during the last week of UCMS or CUS. Only Yue and colleagues performed testing using the FST and OFT both on the first and last day of their 3-week CUS paradigm.

Following chronic stress application, the studies reported findings consistent with depressive-like behaviour. In addition, neurobiological aberrations in the form of cellular as well as humoral immune changes in the blood and in frontolimbic areas were found. It must be noted that Iwata et al.2016) did not reportthese changes following their CUS regime, but in a separate subsection of their study employing acute immobilisation stress. Half the studies showed a significant increase of P2X7R and NLRP3 expression alongside considerable M/Ms activation. Similarly, an upregulation of proinflammatory (NFKB, IL-1 $\beta$, IL-6) and apoptosis-related markers was found. Without exception, P2X7R antagonist application led to a significant attenuation of the UCMS- and CUS-induced neurobiological alterations and depressivelike behaviours. Aricioglu et al. (2009) even reported dosedependent effects of BBG, with $50 \mathrm{mg} / \mathrm{kg}$ being more effective in reducing immobility in the FST than $25 \mathrm{mg} / \mathrm{kg}$. None of the studies reported any side effects of BBG, A-438079 or A-804598 administration.

\section{Discussion and perspectives}

The four studies jointly demonstrate that CUS and UCMS induces depressive-like behaviour in rodents. The observed behavioural aberrations were accompanied by frontal and limbic M/Ms activation and NLRP3-/NFkB-mediated IL-1 $\beta$ increase. Moreover, the administration of a P2X7R antagonist ameliorated the stress effects on a cellular, humoral and behavioural level throughout the different experimental settings. These findings are in line with existing evidence demonstrating that CPSS/CPPS models like CUS, UCMS, CSD, chronic restraint or social isolation lead to NLRP3-/ NFkB-related cytokine release (IL-1 $\beta$, IL-6, TNF- $\alpha$ ) [35, $88,130,133,141]$ and $\mathrm{M} / \mathrm{Ms}$ activation, primarily in limbic structures like the PFC, hippocampus and nucleus accumbens $[90,91,95,130,142-145]$.

In addition, and consistent with studies of NLRP3-, P2X7R- and IL-1 $\beta$ knockout strains [94, 113, 116, 129, 133, 146], chronic $P 2 X 7 R$ antagonist injection led to considerable antidepressive and anti-inflammatory effects in each study. Similar effects were reported by Ribeiro et al. 2019, Catanzaro et al. 2014 and in a subsection of Iwata et al. 2016 in the context of acute stress [67, 94, 147, 148]. Related studies from other immunological research like alcohol-induced 


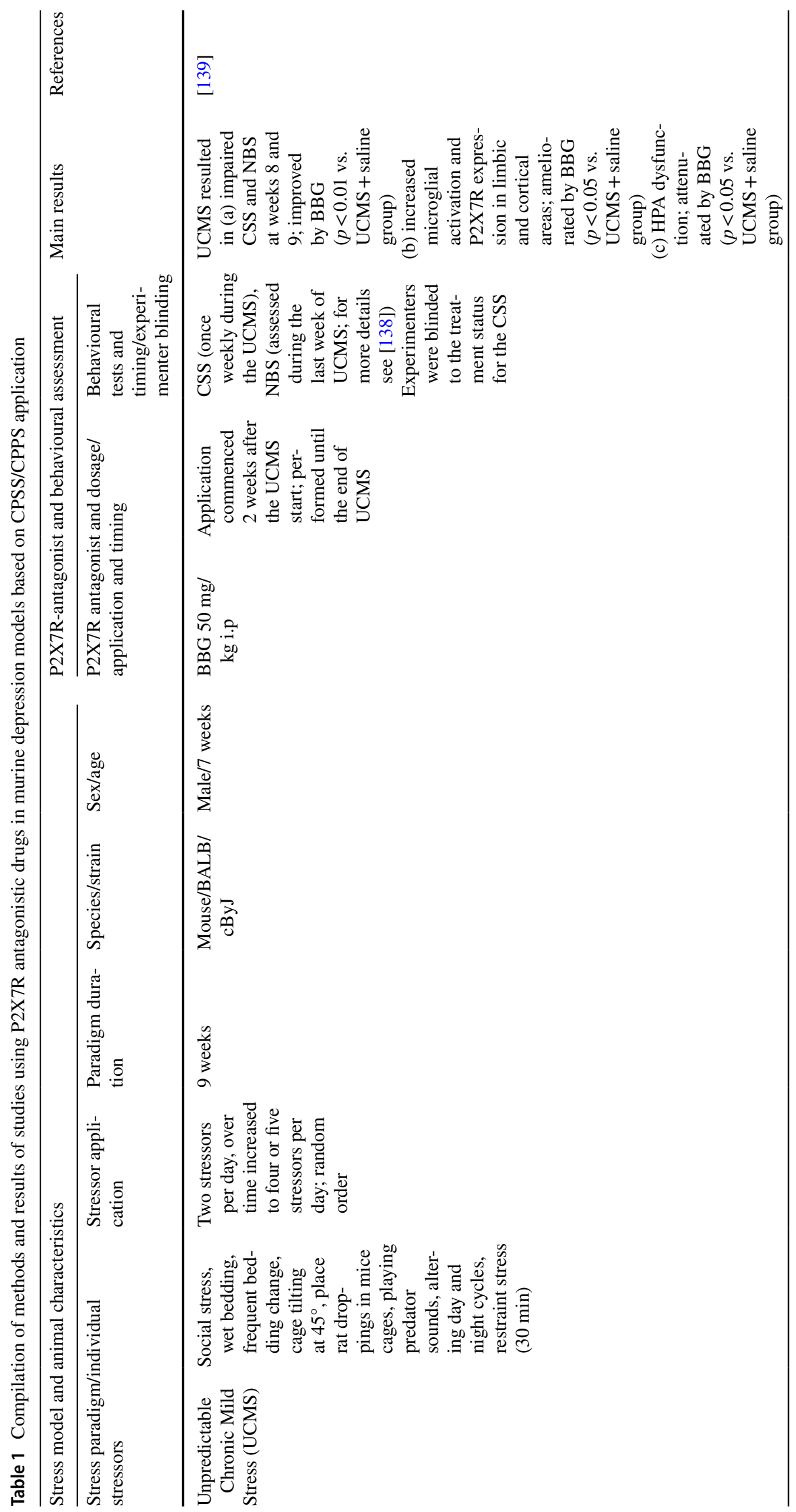




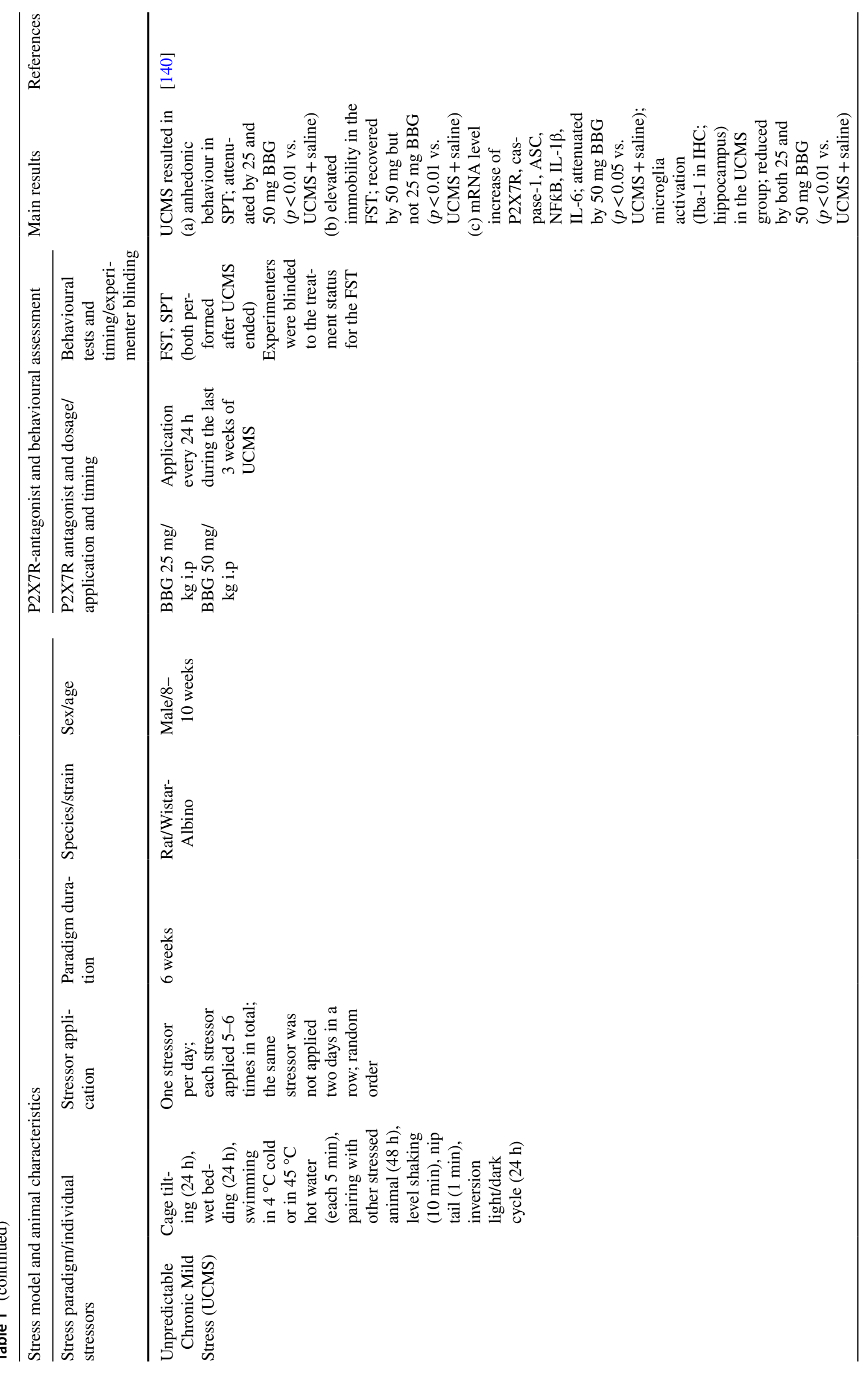




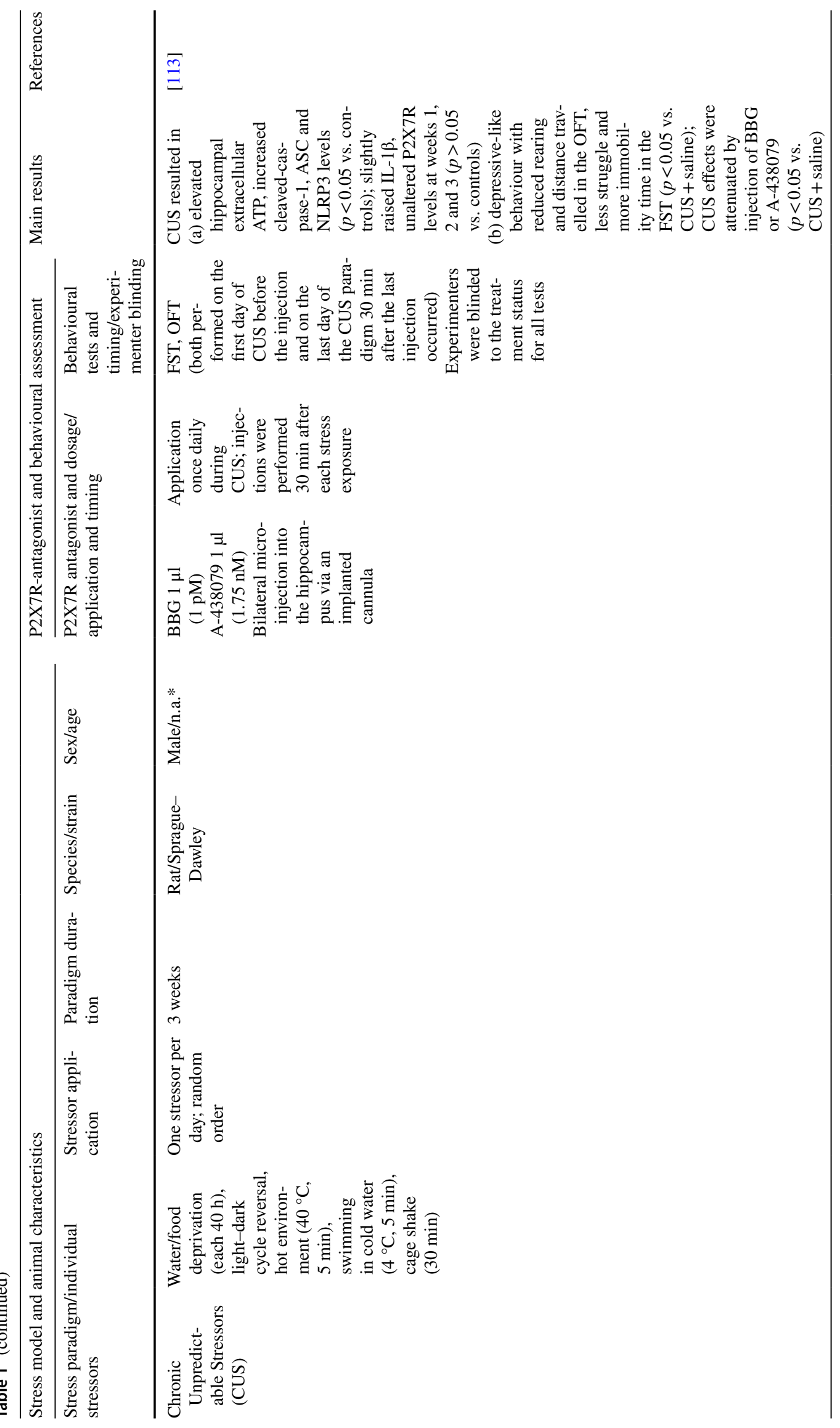







cirrhosis, rheumatoid arthritis and multiple sclerosis demonstrate comparably beneficial and anti-inflammatory properties of P2X7R antagonists [43, 107, 149, 150]. Overall, these results and commonalities with related studies substantiate the upcoming notion of inflammation as an important element in depression genesis [15, 16, 26, 27, 151].

Modelling depression in animals is traditionally based on the application of CPSS/CPPS or certain biological stressors during development, adolescence and adulthood [152]. In the light of three core validity criteria (construct/face/ predictive), each different method can at least model one distinct environmental, neurobiological, or behavioural feature of depression [152, 153]. Although no single approach can resemble all disease factors, chronic, stress-based models like UCMS or CUS are considered the most valid options in reflecting disease complexity overall $[13,54-56]$. However, the models reviewed here mostly lack psychosocial stressors and thus are much more CPPS than CPSS models [25, 154]. In this regard and in line with existing research [155-157], Du Preez et al. [158] previously showed distinct behavioural and neurobiological differences between CPSS, CPPS and combined CPSS/CPPS paradigms in male mice. Repeated saline injections led to anxiety-like behaviour in the OFT and NSFT, increased M/Ms activation and reduced TNF- $\alpha$ serum levels, corticosterone reactivity and hippocampal neuroplasticity. Meanwhile, social isolation resulted in depressive-like behaviour in the SPT as well as FST, increased hippocampal plasticity and elevated serum TNF- $\alpha$, accompanied by decreased IL- $1 \beta$, corticosterone reactivity and M/ Ms density. Intriguingly, the combination of stressors led to yet another phenotype with increased anxiety-like features, reduced serum IL-1 $\beta$ levels and hippocampal plasticity but without significant alterations in corticosterone reactivity and $\mathrm{M} / \mathrm{Ms}$ activation. These findings indicate that the biological and behavioural response strongly depends on the quality and composition of chronic stressors.

Despite the major role of CPSS in depression pathogenesis in humans, no translational studies have so far evaluated the effects of P2X7R antagonists in a CPSS-based depression-model like CSD. To measure stress effects, the reviewed studies used well-established behavioural tests. However, test battery composition was heterogenous and performed based on an endophenotype-driven hypothesis, increasing the risk of constraint and selection. In principle, neurocognitive and behavioural assessment needs to evolve past single test limitations and work towards a comprehensive approach to capture the entirety of psychosocial alterations of a distinct neuropsychiatric phenotype [13, 159]. Though different sentiments exist on how to best assess and class animal models and behaviours [159], the National Institute of Mental Health Research Domain Criteria (RDoC) are a fine-grained matrix interconnecting complex neurobiological aberrations and behavioural domains $[55,153,160]$.
This enables precise, endophenotype-driven modelling of physiological systems involved in the pathogenesis of neuropsychiatric disorders. In addition, due to the interspecies homology within the RDoC domains, findings can be translated from animals to humans and vice versa [13, 14].

On a related note, the studies at hand only used male animals at the verge of adulthood. In neuropsychiatric research, considerable sex effects have been shown for animal behaviour varying by disease state, species and strain [161-163] as well as for the cellular response in general and M/Ms response in particular [163-166]. Moreover, contemporary studies have emphasized the need of a proper age-translation matrix between rodents and human in research $[167,168]$. Future studies using P2X7R antagonists in murine depression models should include both sexes, account for age effects and employ standardized and comprehensive behavioural assessments. In addition to that, the widespread use of imprecise language in reporting behavioural results of neuropsychiatric animal models needs to be improved to reduce the risk of false interpretation primarily in the form of overgeneralisation [13, 153, 169, 170].

Our analysis of the pharmacological properties of the P2X7R antagonists used in the four studies is as follows: $\mathrm{BBG}$, the most used substance, is a non-selective P2X7R antagonist, that penetrates the blood-brain barrier and, aside P2X7R, binds P2X1/2/3/4/5R and voltage-gated sodium channels [43, 108, 171, 172]. In general, antagonist affinity varies depending on the drug itself, the P2Xsubtype, and the species as well as strain [173, 174]. The half maximal inhibitory concentration of BBG for P2X7R in rat and human cells lies between 10 and $200 \mathrm{nM}$, meaning it is up to 50-fold lower than for the other receptors [43, 107, 174]. It even acts 1000-times stronger on P2X7R than on P2X4R [174], making it a semi- rather than a nonselective antagonist. BBG has been used in a variety of research settings revolving around the role of P2X7R and $\mathrm{M} / \mathrm{Ms}$ in neuroinflammation, which overall yielded positive and anti-inflammatory results [43, 175, 176]. However, a lack of in vivo pharmacodynamic analyses in regards to BBG-mediated P2X7R antagonism in the brain has been demonstrated, questioning the overall validity and interpretability of published data [176]. A-804598 and A-438079 can cross the blood-brain barrier and are selective P2X7R antagonists [43, 173, 177]. A-438079 has been used in several inflammatory disease models, causing beneficial effects [178]. However, due to its short biological half-life and limited bioavailability it is considered unsuitable for chronic or clinical application [108, 177, 179]. In contrast, A-804598 has been used in different inflammation models [30, 43, 149, $150,180]$ as well as in acute stress $[147,148]$ and mania models [181]. Based on the limited evidence in the context of neuropsychiatry, the used antagonists can be considered contemporary and appropriate. However, the application 
of antagonists with different pharmacological properties in various stress paradigms allows only mixed receptor blockage along with paradigm-selective behavioural effects to be deducted. Results are, therefore, only partially comparable and must be interpreted with caution [108]. In the future, the attribution of study findings for P2X7R should be reserved for models using highly selective antagonists. Furthermore, studies should take the pharmacological properties, such as P2X7R affinity, into account and favour selective over nonselective drugs [176].

Altogether, we propose a strict commitment to the RDoC and to the newly introduced STRANGE framework (social background, trappability and self-selection, rearing history, acclimation and habituation, natural changes in responsiveness, genetic make-up, experience) in study planning and reporting in translational neuropsychiatry to minimize experimental bias, maximize animal usage, increase animal data quality and impact by enhancing reproducibility and generalizability overall $[55,153,160,182,183]$.

\section{Conclusion}

The four reviewed studies successfully induced depressivelike behaviour, immune changes (NLRP3 assembly, IL-1 $\beta$ level increase, M/Ms activation) and hippocampal neuroplasticity impairment by use of different chronic, psychophysical stress paradigms. P2X7R antagonist application (BBG, A-438079, A-804598) led to an attenuation of the stress-induced neurobiological and behavioural aberrations in all four studies. These findings highlight the potential of P2X7R modulation in chronic stress and depression. However, to advance our understanding of specific P2X7Rrelated effects, methodological refinements are needed. We propose a commitment to the RDoC and the STRANGE framework in study planning and reporting to reduce methodological and pharmacological heterogeneity, minimize bias and increase data validity, reliability, and generalizability. Ultimately, we believe this to be the roadmap to reform animal data impact and improve the life of depressed patients worldwide.

Author contributions IVMH reviewed the scientific literature and wrote the manuscript. CR and LU contributed equally to revision and structuring of the work. JMD reviewed and edited the manuscript and provided essential scientific advice and guidance. The authors thank Jessica Keverne for her help with English language editing.

Funding Open Access funding enabled and organized by Projekt DEAL. IVMH received funding from the Else-Kroener-Fresenius Foundation. IVMH and CR received minor funding from the International Max Planck Research School for Translational Psychiatry. JMD received funding from the German Federal Ministry of Education and Research (IMADAPT, FKZ: 01KU1901) and from the
Marie Skłodowska-Curie innovative training network PurinesDX. LU received a scholarship of the Marie Skłodowska-Curie innovative training network PurinesDX.

Availability of data and materials (data transparency) To identify suitable research concerning the effects of $\mathrm{P} 2 \mathrm{X} 7$ receptor antagonists on chronic stress effects in animal models, IVMH searched the electronic database PubMed using three broad keyword combinations: ( $P 2 X 7 R$ antagonist) $A N D$ (chronic stress) $\longrightarrow 13$ results; $(P 2 X 7 R$ antagonist $)$ $A N D$ (stress) $\longrightarrow 41$ results; $(P 2 X 7$ antagonist) $A N D$ (stress $) \longrightarrow 97$ results. Out of these available studies, only 4 met the inclusion criteria (i.e., chronic psychosocial or psychophysical stress model of depressive-like behaviour in animals; iterative $\mathrm{P} 2 \mathrm{X} 7$ antagonist application). These publications are reviewed in this article.

Code availability (software application or custom code) Not applicable.

\section{Declarations}

Conflict of interest The authors declare that the research was conducted in the absence of any financial interest, which could be viewed as a potential conflict of interest.

Studies involving humans and/or animals Not applicable.

Ethical approval Not applicable.

Consent to participate Not applicable.

Consent for publication Not applicable.

Open Access This article is licensed under a Creative Commons Attribution 4.0 International License, which permits use, sharing, adaptation, distribution and reproduction in any medium or format, as long as you give appropriate credit to the original author(s) and the source, provide a link to the Creative Commons licence, and indicate if changes were made. The images or other third party material in this article are included in the article's Creative Commons licence, unless indicated otherwise in a credit line to the material. If material is not included in the article's Creative Commons licence and your intended use is not permitted by statutory regulation or exceeds the permitted use, you will need to obtain permission directly from the copyright holder. To view a copy of this licence, visit http://creativecommons.org/licenses/by/4.0/.

\section{References}

1. Pedersen SH et al (2001) ICD-10 criteria for depression in general practice. J Affect Disord 65(2):191-194

2. Depression and Other Common Mental Disorders: Global Health Estimates (2017) Licence: CC BY-NC-SA 3.0 IGO. World Health Organization, Genva

3. Van de Velde S, Bracke P, Levecque K (2010) Gender differences in depression in 23 European countries. Cross-national variation in the gender gap in depression. Soc Sci Med 71(2):305-313

4. James SL et al (2018) Global, regional, and national incidence, prevalence, and years lived with disability for 354 diseases and injuries for 195 countries and territories, 1990-2017: a systematic analysis for the Global Burden of Disease Study 2017. Lancet 392(10159):1789-1858 
5. König H, König HH, Konnopka A (2020) The excess costs of depression: a systematic review and meta-analysis. Epidemiol Psychiatr Sci 29:e30

6. Richards D (2011) Prevalence and clinical course of depression: a review. Clin Psychol Rev 31(7):1117-1125

7. Kessler R (2012) The costs of depression. Psychiatr Clin North Am 35:1-14

8. Kraus C et al (2019) Prognosis and improved outcomes in major depression: a review. Transl Psychiatry 9(1):127

9. Cuijpers P, Stringaris A, Wolpert M (2020) Treatment outcomes for depression: challenges and opportunities. Lancet Psychiatry 7(11):925-927

10. Spijker J et al (2002) Duration of major depressive episodes in the general population: results from The Netherlands Mental Health Survey and Incidence Study (NEMESIS). Br J Psychiatry 181:208-213

11. Eaton WW et al (2008) Population-based study of first onset and chronicity in major depressive disorder. Arch Gen Psychiatry 65(5):513-520

12. Nübel J et al (2020) Persistent depressive disorder across the adult lifespan: results from clinical and population-based surveys in Germany. BMC Psychiatry 20(1):58

13. Bale TL et al (2019) The critical importance of basic animal research for neuropsychiatric disorders. Neuropsychopharmacology 44(8):1349-1353

14. Anderzhanova E, Kirmeier T, Wotjak CT (2017) Animal models in psychiatric research: the $\mathrm{RDoC}$ system as a new framework for endophenotype-oriented translational neuroscience. Neurobiol Stress 7:47-56

15. Wohleb ES et al (2016) Integrating neuroimmune systems in the neurobiology of depression. Nat Rev Neurosci 17(8):497-511

16. Miller AH, Raison CL (2016) The role of inflammation in depression: from evolutionary imperative to modern treatment target. Nat Rev Immunol 16(1):22-34

17. Rial D et al (2016) Depression as a glial-based synaptic dysfunction. Front Cell Neurosci. https://doi.org/10.3389/fncel.2015. 00521

18. Singhal G, Baune BT (2017) Microglia: an interface between the loss of neuroplasticity and depression. Front Cell Neurosci. https://doi.org/10.3389/fncel.2017.00270

19. Liu RT (2017) Childhood adversities and depression in adulthood: current findings and future directions. Clin Psychol 24(2):140-153

20. Plieger $\mathrm{T}$ et al (2015) Life stress as potential risk factor for depression and burnout. Burn Res 2(1):19-24

21. Hammen C (2005) Stress and depression. Annu Rev Clin Psychol 1:293-319

22. Hammen C (2018) Risk factors for depression: an autobiographical review. Annu Rev Clin Psychol 14(1):1-28

23. Hazel NA et al (2008) Early childhood adversity and adolescent depression: the mediating role of continued stress. Psychol Med 38(4):581-589

24. Hammen CL (2015) Stress and depression: old questions, new approaches. Curr Opin Psychol 4:80-85

25. Pryce CR, Fuchs E (2016) Chronic psychosocial stressors in adulthood: studies in mice, rats and tree shrews. Neurobiol Stress 6:94-103

26. Deng S-L, Chen J-G, Wang F (2020) Microglia: a central player in depression. Curr Med Sci 40(3):391-400

27. Yirmiya R, Rimmerman N, Reshef R (2015) Depression as a microglial disease. Trends Neurosci 38(10):637-658

28. Stokes L, Spencer SJ, Jenkins TA (2015) Understanding the role of P2X7 in affective disorders-are glial cells the major players? Front Cell Neurosci 9:258-258
29. Janks L, Sharma CVR, Egan TM (2018) A central role for P2X7 receptors in human microglia. J Neuroinflammation 15(1):325

30. He Y et al (2017) The role of microglial P2X7: modulation of cell death and cytokine release. J Neuroinflammation 14(1):135

31. Kreisel $\mathrm{T}$ et al (2014) 7. The role of microglia in stress-induced depression. Brain Behav Immun 40:e2-e3

32. Frick LR, Williams K, Pittenger C (2013) Microglial dysregulation in psychiatric disease. Clin Dev Immunol 2013:608654

33. Illes P, Verkhratsky A, Tang Y (2020) Pathological ATPergic signaling in major depression and bipolar disorder. Front Mol Neurosci. https://doi.org/10.3389/fnmol.2019.00331

34. Ribeiro DE et al (2019) P2X7 receptor signaling in stress and depression. Int J Mol Sci 20(11):2778

35. Wei L et al (2018) ATP-activated P2X7 receptor in the pathophysiology of mood disorders and as an emerging target for the development of novel antidepressant therapeutics. Neurosci Biobehav Rev 87:192-205

36. Di Virgilio F et al (2017) The P2X7 receptor in infection and inflammation. Immunity 47(1):15-31

37. Di Virgilio F (2007) Liaisons dangereuses: P2X(7) and the inflammasome. Trends Pharmacol Sci 28(9):465-472

38. Wang D et al (2020) P2X7 receptor mediates NLRP3 inflammasome activation in depression and diabetes. Cell Biosci 10(1):28

39. Bartoli F et al (2020) Purinergic signaling and related biomarkers in depression. Brain Sci 10(3):160

40. Jacob F et al (2013) Purinergic signaling in inflammatory cells: $\mathrm{P} 2$ receptor expression, functional effects, and modulation of inflammatory responses. Purinergic Signal 9(3):285-306

41. Czamara D, Müller-Myhsok B, Lucae S (2017) The P2RX7 polymorphism rs2230912 is associated with depression: a meta-analysis. Progr Neuropsychopharmacol Biol Psychiatry 82:272-277

42. Lucae $\mathrm{S}$ et al (2006) P2RX7, a gene coding for a purinergic ligand-gated ion channel, is associated with major depressive disorder. Hum Mol Genet 15(16):2438-2445

43. Andrejew R et al (2020) The P2X7 receptor: central hub of brain diseases. Front Mol Neurosci 13:124

44. Soronen P et al (2011) P2RX7 gene is associated consistently with mood disorders and predicts clinical outcome in three clinical cohorts. Am J Med Genet B Neuropsychiatr Genet 156(4):435-447

45. Metzger MW et al (2017) Genetically dissecting P2rx7 expression within the central nervous system using conditional humanized mice. Purinergic Signal 13(2):153-170

46. Metzger MW et al (2017) Heterozygosity for the mood disorderassociated variant Gln460Arg alters P2X7 receptor function and sleep quality. J Neurosci 37(48):11688-11700

47. Deussing JM, Arzt E (2018) P2X7 receptor: a potential therapeutic target for depression? Trends Mol Med 24(9):736-747

48. Bhattacharya A, Jones DNC (2018) Emerging role of the P2X7NLRP3-IL1 $\beta$ pathway in mood disorders. Psychoneuroendocrinology 98:95-100

49. Bhattacharya A, Ceusters M (2020) Targeting neuroinflammation with brain penetrant $\mathrm{P} 2 \mathrm{X} 7$ antagonists as novel therapeutics for neuropsychiatric disorders. Neuropsychopharmacology 45(1):234-235

50. Rech J et al (2016) The evolution of P2X7 antagonists with a focus on CNS indications. Bioorgan Med Chem Lett $26: 3838-3845$

51. Jiang L-H et al (2020) Targeting the P2X7 receptor in microglial cells to prevent brain inflammation. Neural Regen Res 15(7):1245-1246

52. US National Library of Medicine (2019) Clinical trials.gov: antidepressant trial with P2X7 antagonist JNJ-54175446 (ATP) [cited 06.11.2020]. https://clinicaltrials.gov/ct2/show/NCT04 116606 
53. US National Library of Medicine (2019) - Clinical trials.gov: a study of JNJ-61393215 in the treatment of depression [cited 06.11.2020]. https://clinicaltrials.gov/ct2/show/NCT04080752

54. Czéh B et al (2016) Animal models of major depression and their clinical implications. Prog Neuropsychopharmacol Biol Psychiatry 64:293-310

55. Söderlund J, Lindskog M (2018) Relevance of rodent models of depression in clinical practice: can we overcome the obstacles in translational neuropsychiatry? Int J Neuropsychopharmacol 21(7):668-676

56. Willner P (2017) The chronic mild stress (CMS) model of depression: history, evaluation and usage. Neurobiol Stress 6:78-93

57. Dean J, Keshavan M (2017) The neurobiology of depression: an integrated view. Asian J Psychiatr 27:101-111

58. Szabo S et al (2017) "Stress" is 80 years old: from hans selye original paper in 1936 to recent advances in GI ulceration. Curr Pharm Des 23(27):4029-4041

59. Selye H (1936) A syndrome produced by diverse nocuous agents. Nature 138(3479):32-32

60. Tennant C (2002) Life events, stress and depression: a review of recent findings. Aust N Z J Psychiatry 36(2):173-182

61. Humphreys KL et al (2020) Child maltreatment and depression: a meta-analysis of studies using the childhood trauma questionnaire. Child Abuse Neglect 102:104361

62. Nelson J et al (2017) Childhood maltreatment and characteristics of adult depression: meta-analysis. Br J Psychiatry 210(2):96-104

63. Assari S, Lankarani MM (2016) Stressful life events and risk of depression 25 years later: race and gender differences. Front Public Health. https://doi.org/10.3389/fpubh.2016.00049

64. Li Yue ZD, Yinghao L, Tianqiang H (2016) Meta-analysis of the relationship between life events and depression in adolescents. $\mathrm{J}$ Pediatr Care 2:1

65. Culverhouse RC et al (2018) Collaborative meta-analysis finds no evidence of a strong interaction between stress and 5-HTTLPR genotype contributing to the development of depression. Mol Psychiatry 23(1):133-142

66. Tibubos AN et al (2020) Frequency of stressful life events and associations with mental health and general subjective health in the general population. J Public Health. https://doi.org/10.1007/ s10389-020-01204-3

67. Iwata M, Ota KT, Duman RS (2013) The inflammasome: pathways linking psychological stress, depression, and systemic illnesses. Brain Behav Immun 31:105-114

68. Lee C-H, Giuliani F (2019) The role of inflammation in depression and fatigue. Front Immunol 10:1696-1696

69. Réus $\mathrm{G}$ et al (2015) The role of inflammation and microglial activation in the pathophysiology of psychiatric disorders. Neuroscience 300:141-154

70. Danese A et al (2007) Childhood maltreatment predicts adult inflammation in a life-course study. Proc Natl Acad Sci USA 104(4):1319-1324

71. Dowlati Y et al (2010) A meta-analysis of cytokines in major depression. Biol Psychiat 67(5):446-457

72. Kappelmann $\mathrm{N}$ et al (2020) Dissecting the association between inflammation, metabolic dysregulation, and specific depressive symptoms: a genetic correlation and 2-sample mendelian randomization study. JAMA Psychiat. https://doi.org/10.1001/jamap sychiatry.2020.3436

73. Kim Y-K et al (2007) Cytokine imbalance in the pathophysiology of major depressive disorder. Prog Neuropsychopharmacol Biol Psychiatry 31(5):1044-1053

74. Köhler CA et al (2017) Peripheral cytokine and chemokine alterations in depression: a meta-analysis of 82 studies. Acta Psychiatr Scand 135(5):373-387
75. Beydoun MA et al (2020) Systemic inflammation is associated with depressive symptoms differentially by sex and race: a longitudinal study of urban adults. Mol Psychiatry 25(6):1286-1300

76. Miller AH, Maletic V, Raison CL (2009) Inflammation and its discontents: the role of cytokines in the pathophysiology of major depression. Biol Psychiat 65(9):732-741

77. Hiles SA et al (2012) A meta-analysis of differences in IL-6 and IL-10 between people with and without depression: exploring the causes of heterogeneity. Brain Behav Immun 26(7):1180-1188

78. Zou W, Feng R, Yang Y (2018) Changes in the serum levels of inflammatory cytokines in antidepressant drug-naïve patients with major depression. PLoS ONE 13(6):e0197267-e0197267

79. Anderson RJ et al (2001) The prevalence of comorbid depression in adults with diabetes. A meta-analysis. Diabetes Care 24(6):1069-1078

80. Du YJ et al (2013) Airway inflammation and hypothalamic-pituitary-adrenal axis activity in asthmatic adults with depression. J Asthma 50(3):274-281

81. Laake JP et al (2014) The association between depressive symptoms and systemic inflammation in people with type 2 diabetes: findings from the South London Diabetes Study. Diabetes Care 37(8):2186-2192

82. Sanna L et al (2014) Atopic disorders and depression: findings from a large, population-based study. J Affect Disord 155:261-265

83. Alcocer-Gómez E et al (2014) NLRP3 inflammasome is activated in mononuclear blood cells from patients with major depressive disorder. Brain Behav Immun 36:111-117

84. Monif $\mathrm{M}$ et al (2009) The P2X7 receptor drives microglial activation and proliferation: a trophic role for P2X7R pore. J Neurosci 29(12):3781-3791

85. Bianco $\mathrm{F}$ et al (2006) A role for $\mathrm{P} 2 \mathrm{X} 7$ in microglial proliferation. J Neurochem 99(3):745-758

86. Stein DJ et al (2017) Microglial over-activation by social defeat stress contributes to anxiety- and depressive-like behaviors. Front Behav Neurosci. https://doi.org/10.3389/fnbeh.2017. 00207

87. Hinwood M et al (2012) Evidence that microglia mediate the neurobiological effects of chronic psychological stress on the medial prefrontal cortex. Cereb Cortex 22(6):1442-1454

88. Wang Y-L et al (2018) Microglial activation mediates chronic mild stress-induced depressive- and anxiety-like behavior in adult rats. J Neuroinflammation 15(1):21

89. Walker F, Nilsson M, Jones K (2013) Acute and chronic stressinduced disturbances of microglial plasticity, phenotype and function. Curr Drug Targets 14:1262-1276

90. Wohleb ES et al (2013) Stress-induced recruitment of bone marrow-derived monocytes to the brain promotes anxiety-like behavior. J Neurosci 33(34):13820-13833

91. Wohleb ES et al (2014) Re-establishment of anxiety in stresssensitized mice is caused by monocyte trafficking from the spleen to the brain. Biol Psychiatry 75(12):970-981

92. Wohleb ES et al (2012) Peripheral innate immune challenge exaggerated microglia activation, increased the number of inflammatory CNS macrophages, and prolonged social withdrawal in socially defeated mice. Psychoneuroendocrinology 37(9):1491-1505

93. Frank MG et al (2007) Microglia serve as a neuroimmune substrate for stress-induced potentiation of CNS pro-inflammatory cytokine responses. Brain Behav Immun 21(1):47-59

94. Iwata $\mathrm{M}$ et al (2016) Psychological stress activates the inflammasome via release of adenosine triphosphate and stimulation of the purinergic type 2 X7 receptor. Biol Psychiat 80(1):12-22

95. Calcia MA et al (2016) Stress and neuroinflammation: a systematic review of the effects of stress on microglia and the implications for mental illness. Psychopharmacology 233(9):1637-1650 
96. Setiawan E et al (2015) Role of translocator protein density, a marker of neuroinflammation, in the brain during major depressive episodes. JAMA Psychiat 72(3):268-275

97. Holmes SE et al (2018) Elevated translocator protein in anterior cingulate in major depression and a role for inflammation in suicidal thinking: a positron emission tomography study. Biol Psychiat 83(1):61-69

98. Richards EM et al (2018) PET radioligand binding to translocator protein (TSPO) is increased in unmedicated depressed subjects. EJNMMI Res 8(1):57

99. Rupprecht $\mathrm{R}$ et al (2010) Translocator protein (18 kDa) (TSPO) as a therapeutic target for neurological and psychiatric disorders. Nat Rev Drug Discov 9(12):971-988

100. Lehmann ML et al (2016) Social defeat induces depressive-like states and microglial activation without involvement of peripheral macrophages. J Neuroinflammation 13(1):224

101. Torres-Platas SG et al (2014) Evidence for increased microglial priming and macrophage recruitment in the dorsal anterior cingulate white matter of depressed suicides. Brain Behav Immun 42:50-59

102. Suzuki H et al (2019) Suicide and microglia: recent findings and future perspectives based on human studies. Front Cell Neurosci 13:31-31

103. Baharikhoob P, Kolla NJ (2020) Microglial dysregulation and suicidality: a stress-diathesis perspective. Front Psychiatry. https://doi.org/10.3389/fpsyt.2020.00781

104. Steiner J et al (2008) Immunological aspects in the neurobiology of suicide: elevated microglial density in schizophrenia and depression is associated with suicide. J Psychiatr Res 42(2):151-157

105. Wang $\mathbf{J}$ et al (2020) Molecular mechanisms of glial cells related signaling pathways involved in the neuroinflammatory response of depression. Mediators Inflamm 2020:3497920

106. Giuliani A, Sarti A, Di Virgilio F (2018) Extracellular nucleotides and nucleosides as signalling molecules. Immunol Lett 205:16-24

107. Burnstock G, Verkhratsky A (2012) Purinergic signalling and the nervous system. In: Receptors for Purines and Pyrimidines, 1st edn, Chap 5. Springer Publishing, Berlin/Heidelberg, p 119. https://doi.org/10.1007/978-3-642-28863-0

108. Savio LEB et al (2018) The P2X7 receptor in inflammatory diseases: angel or demon? Front Pharmacol 9:52-52

109. Adinolfi $\mathrm{E}$ et al (2018) The P2X7 receptor: a main player in inflammation. Biochem Pharmacol 151:234-244

110. Lord B et al (2015) A novel radioligand for the ATP-gated ion channel P2X7: [3H] JNJ-54232334. Eur J Pharmacol 765:551-559

111. Jacobson KA, Müller CE (2016) Medicinal chemistry of adenosine, P2Y and P2X receptors. Neuropharmacology 104:31-49

112. Illes $P$ et al (2020) Regulation of microglial functions by purinergic mechanisms in the healthy and diseased CNS. Cells 9(5): 1108

113. Yue $\mathrm{N}$ et al (2017) Activation of $\mathrm{P} 2 \mathrm{X} 7$ receptor and NLRP3 inflammasome assembly in hippocampal glial cells mediates chronic stress-induced depressive-like behaviors. J Neuroinflammation 14(1):102

114. Musazzi L et al (2010) Acute stress increases depolarizationevoked glutamate release in the rat prefrontal/frontal cortex: the dampening action of antidepressants. PLoS ONE $5(1): \mathrm{e} 8566$

115. Mayhew J et al (2018) Purinergic modulation of glutamate transmission: an expanding role in stress-linked neuropathology. Neurosci Biobehav Rev 93:26-37

116. Alcocer-Gómez E et al (2016) Stress-induced depressive behaviors require a functional NLRP3 inflammasome. Mol Neurobiol 53(7):4874-4882
117. Di Virgilio F, Schmalzing G, Markwardt F (2018) The elusive P2X7 macropore. Trends Cell Biol 28(5):392-404

118. Vénéreau E, Ceriotti C, Bianchi ME (2015) DAMPs from cell death to new life. Front Immunol 6:422-422

119. Pippel A et al (2017) Localization of the gate and selectivity filter of the full-length P2X7 receptor. Proc Natl Acad Sci U S A 114(11):E2156-e2165

120. Harkat $\mathrm{M}$ et al (2017) On the permeation of large organic cations through the pore of ATP-gated P2X receptors. Proc Natl Acad Sci 114(19):E3786-E3795

121. He $\mathrm{Y}$ et al (2016) NEK7 is an essential mediator of NLRP3 activation downstream of potassium efflux. Nature 530(7590):354-357

122. Kaufmann FN et al (2017) NLRP3 inflammasome-driven pathways in depression: clinical and preclinical findings. Brain Behav Immun 64:367-383

123. Erb L et al (2006) P2 receptors: intracellular signaling. Pflugers Arch 452(5):552-562

124. Dinarello CA (2013) Overview of the interleukin-1 family of ligands and receptors. Semin Immunol 25(6):389-393

125. Dinarello CA (2004) The IL-1 family: the role of IL-1 and IL-18 in inflammation. In: van den Berg WB, Miossec P (eds) Cytokines and Joint Injury. Birkhäuser Basel, Basel, pp 29-67

126. Velasquez S, Rappaport J (2016) Inflammasome activation in major depressive disorder: a pivotal linkage between psychological stress, purinergic signaling, and the kynurenine pathway. Biol Psychiatry 80(1):4-5

127. Delpech J-C et al (2015) Microglia in neuronal plasticity: influence of stress. Neuropharmacology 96:19-28

128. Colyn L et al (2019) Chronic social defeat stress induces sustained synaptic structural changes in the prefrontal cortex and amygdala. Behav Brain Res 373:112079

129. Basso A et al (2008) Behavioral profile of $\mathrm{P} 2 \mathrm{X} 7$ receptor knockout mice in animal models of depression and anxiety: Relevance for neuropsychiatric disorders. Behav Brain Res 198:83-90

130. Pan $Y$ et al (2014) Microglial NLRP3 inflammasome activation mediates IL-1 $\beta$-related inflammation in prefrontal cortex of depressive rats. Brain Behav Immun 41:90-100

131. Zhang $Y$ et al (2015) NLRP3 inflammasome mediates chronic mild stress-induced depression in mice via neuroinflammation. Int J Neuropsychopharmacol 18(8):pyv006

132. Park H-J et al (2015) IL-4 inhibits IL-1 $\beta$-induced depressivelike behavior and central neurotransmitter alterations. Mediat Inflam 2015:941413

133. Goshen I et al (2008) Brain interleukin-1 mediates chronic stress-induced depression in mice via adrenocortical activation and hippocampal neurogenesis suppression. Mol Psychiatry 13(7):717-728

134. Kaster $M$ et al (2011) Depressive-like behavior induced by tumor necrosis factor-I in mice. Neuropharmacology 62:419-426

135. Karson A et al (2012) Chronic administration of infliximab (TNF-Alpha Inhibitor) decreases depression and anxiety-like behaviour in rat model of chronic mild stress. Basic Clin Pharmacol Toxicol 112:335-340

136. Zhang JC et al (2017) Blockade of interleukin-6 receptor in the periphery promotes rapid and sustained antidepressant actions: a possible role of gut-microbiota-brain axis. Transl Psychiatry 7(5):e1138-e1138

137. Chourbaji S et al (2006) IL-6 knockout mice exhibit resistance to stress-induced development of depression-like behaviors. Neurobiol Dis 23:587-594

138. Deacon RMJ (2006) Assessing nest building in mice. Nat Protoc $1(3): 1117-1119$ 
139. Farooq RK et al (2018) A P2X7 receptor antagonist reverses behavioural alterations, microglial activation and neuroendocrine dysregulation in an unpredictable chronic mild stress (UCMS) model of depression in mice. Psychoneuroendocrinology 97:120-130

140. Aricioglu F et al (2019) Antidepressant-like effects induced by chronic blockade of the purinergic $2 \times 7$ receptor through inhibition of non-like receptor protein 1 inflammasome in chronic unpredictable mild stress model of depression in rats. Clin Psychopharmacol Neurosci 17(2):261-272

141. Milenkovic VM et al (2019) The role of chemokines in the pathophysiology of major depressive disorder. Int J Mol Sci 20(9):2283

142. Khan AR et al (2020) Stress-induced morphological, cellular and molecular changes in the brain-lessons learned from the chronic mild stress model of depression. Cells 9(4):1026

143. Walker FR, Nilsson M, Jones K (2013) Acute and chronic stressinduced disturbances of microglial plasticity, phenotype and function. Curr Drug Targets 14(11):1262-1276

144. Troubat $\mathrm{R}$ et al (2021) Neuroinflammation and depression: a review. Eur J Neurosci. https://doi.org/10.1111/ejn.14720

145. Kreisel $\mathrm{T}$ et al (2014) Dynamic microglial alterations underlie stress-induced depressive-like behavior and suppressed neurogenesis. Mol Psychiatry 19(6):699-709

146. Boucher AA et al (2011) Resilience and reduced c-Fos expression in $\mathrm{P} 2 \mathrm{X} 7$ receptor knockout mice exposed to repeated forced swim test. Neuroscience 189:170-177

147. Ribeiro DE et al (2019) Antidepressant-like effect induced by $\mathrm{P} 2 \mathrm{X} 7$ receptor blockade in FSL rats is associated with BDNF signalling activation. J Psychopharmacol 33(11):1436-1446

148. Catanzaro JM et al (2014) The impact of the P2X7 receptor antagonist A- 804598 on neuroimmune and behavioral consequences of stress. Behav Pharmacol 25(5-6):582-598

149. Freire D et al (2019) P2X7 receptor antagonist A804598 inhibits inflammation in brain and liver in C57BL/6 J mice exposed to chronic ethanol and high fat diet. J Neuroimmune Pharmacol 14(2):263-277

150. Liu $Y$ et al (2020) The P2X7 receptor (P2X7R)-specific antagonist A804598 inhibits inflammatory reaction in human fibroblastlike synoviocytes. Am J Transl Res 12(1):45-53

151. Prinz M, Priller J (2014) Microglia and brain macrophages in the molecular age: from origin to neuropsychiatric disease. Nat Rev Neurosci 15(5):300-312

152. Planchez B, Surget A, Belzung C (2019) Animal models of major depression: drawbacks and challenges. J Neural Transm 126(11):1383-1408

153. Gururajan A et al (2019) The future of rodent models in depression research. Nat Rev Neurosci 20(11):686-701

154. Pryce CR, Fuchs E (2017) Editorial: stressors in animals and humans-practical issues and limitations. Neurobiol Stress 6:1-2

155. Finnell JE et al (2017) Physical versus psychological social stress in male rats reveals distinct cardiovascular, inflammatory and behavioral consequences. PLoS ONE 12(2):e0172868-e0172868

156. Kavushansky A et al (2009) Physical stress differs from psychosocial stress in the pattern and time-course of behavioral responses, serum corticosterone and expression of plasticityrelated genes in the rat. Stress (Amsterdam, Netherlands) $12: 412-425$

157. Singewald G et al (2009) Effect of chronic psychosocial stressinduced by subordinate colony (CSC) housing on brain neuronal activity patterns in mice. Stress (Amsterdam, Netherlands) 12:58-69

158. Du Preez A et al (2020) The type of stress matters: repeated injection and permanent social isolation stress in male mice have a differential effect on anxiety- and depressive-like behaviours, and associated biological alterations. Transl Psychiatry 10(1):325
159. Stephan M, Volkmann P, Rossner M (2019) Assessing behavior and cognition in rodents, nonhuman primates, and humans: where are the limits of translation? Dialogues Clin Neurosci 21:249-259

160. Woody ML, Gibb BE (2015) Integrating NIMH research domain criteria (RDoC) into depression research. Curr Opin Psychol $4: 6-12$

161. van Doeselaar L et al (2020) Chronic social defeat stress in female mice leads to sex-specific behavioral and neuroendocrine effects. Stress. https://doi.org/10.1080/10253890.2020.1864319

162. Kokras N, Dalla C (2014) Sex differences in animal models of psychiatric disorders. Br J Pharmacol 171(20):4595-4619

163. Yanguas-Casás N (2020) Physiological sex differences in microglia and their relevance in neurological disorders. Neuroimmunol Neuroinflamm 7(1):13-22

164. Bollinger JL, Bergeon Burns CM, Wellman CL (2016) Differential effects of stress on microglial cell activation in male and female medial prefrontal cortex. Brain Behav Immunity 52:88-97

165. Bekhbat M, Neigh GN (2018) Sex differences in the neuroimmune consequences of stress: focus on depression and anxiety. Brain Behav Immun 67:1-12

166. VanRyzin JW, Pickett LA, McCarthy MM (2018) Microglia: driving critical periods and sexual differentiation of the brain. Dev Neurobiol 78(6):580-592

167. Dutta $S$, Sengupta $P$ (2016) Men and mice: relating their ages. Life Sci 152:244-248

168. Sengupta $P(2013)$ The laboratory rat: relating its age with human's. Int J Prev Med 4(6):624-630

169. Parikh M (2015) Subject selection bias in animal studies. Curr Sci 109:678-679

170. Pannucci CJ, Wilkins EG (2010) Identifying and avoiding bias in research. Plast Reconstr Surg 126(2):619-625

171. Jo S, Bean BP (2011) Inhibition of neuronal voltage-gated sodium channels by brilliant blue G. Mol Pharmacol 80(2):247-257

172. Bo X et al (2003) Pharmacological and biophysical properties of the human P2X5 receptor. Mol Pharmacol 63(6):1407-1416

173. Donnelly-Roberts DL et al (2009) Mammalian P2X7 receptor pharmacology: comparison of recombinant mouse, rat and human P2X7 receptors. Br J Pharmacol 157(7):1203-1214

174. Jiang LH et al (2000) Brilliant blue G selectively blocks ATPgated rat P2X(7) receptors. Mol Pharmacol 58(1):82-88

175. Wang $\mathrm{W}$ et al (2020) Brilliant blue $\mathrm{G}$ attenuates neuro-inflammation via regulating MAPKs and NF-\&kappa;B signaling pathways in lipopolysaccharide-induced BV2 microglia cells. Exp Ther Med 20(5):116

176. Bhattacharya A, Biber K (2016) The microglial ATP-gated ion channel P2X7 as a CNS drug target. Glia 64(10):1772-1787

177. Nelson DW et al (2006) Structure-activity relationship studies on a series of novel, substituted 1-benzyl-5-phenyltetrazole P2X7 antagonists. J Med Chem 49(12):3659-3666

178. Burnstock G, Knight GE (2018) The potential of P2X7 receptors as a therapeutic target, including inflammation and tumour progression. Purinergic Signall 14(1):1-18

179. Díaz-Hernández M et al (2009) Altered P2X7-receptor level and function in mouse models of Huntington's disease and therapeutic efficacy of antagonist administration. Faseb J 23(6):1893-1906

180. Fabbrizio $\mathrm{P}$ et al (2017) P2X7 receptor activation modulates autophagy in SOD1-G93A mouse microglia. Front Cell Neurosci $11: 249$

181. Gubert $\mathrm{C}$ et al (2016) Role of $\mathrm{P} 2 \mathrm{X} 7$ receptor in an animal model of mania induced by D-AMPhetamine. Mol Neurobiol 53(1):611-620 
182. Rutz C, Webster MM (2021) Ethology adopts the STRANGE framework for animal behaviour research, to improve reporting standards. Ethology 127(2):99-101
183. Webster MM, Rutz C (2020) How STRANGE are your study animals? Nature 582(7812):337-340 